\title{
THERMODYNAMIC CHARACTERISTICS OF THREE-DIMENSIONAL ISING-LIKE SYSTEMS AS FUNCTIONS OF MICROSCOPIC PARAMETERS. THE $\rho^{6}$ MODEL APPROXIMATION
}

\author{
I. V. Pylyuk \\ Institute for Condensed Matter Physics of the National Academy of Sciences of Ukraine \\ 1 Svientsitskii Str., Lviv, UA-79011, Ukraine
}

(Received August 30, 2001)

\begin{abstract}
The microscopic approach to calculating and investigating the three-dimensional (3D) Ising-like system thermodynamics above and below the phase transition temperature $T_{c}$ is developed using the collective variables method. Mathematical description is performed within the framework of the higher non-Gaussian approximation $\left(\rho^{6}\right.$ model $)$ taking into account the corrections to scaling. A nonuniversal factor depending on microscopic parameters of the system is singled out in the expressions for leading critical amplitudes and correction-to-scaling amplitudes. Numerical estimates of the critical region size, phase transition temperature as well as the graphs of temperature dependences of entropy and other thermodynamic characteristics near $T_{c}$ are given for various values of effective radius of the exponentially decreasing interaction potential. The variation of the free energy of the system at the phase transition point, average spin moment, and specific heat with increasing ratio of the potential effective radius to the simple cubic lattice constant is traced. The results of calculations and their comparison with the other authors' data show that the $\rho^{6}$ model provides a more adequate quantitative description of the critical behaviour of a 3D Ising-like system than the $\rho^{4}$ model.
\end{abstract}

Key words: Ising model, phase transitions, critical behaviour, thermodynamics, corrections to scaling.

PACS number(s): 05.50.+q, 05.70.Ce, 64.60.Fr, 75.10.Hk

\section{INTRODUCTION}

A central problem in statistical physics is the description of phase transitions and critical phenomena, i.e., the construction of a microscopic theory of phase transitions. Intensive studies of phase transitions have made it possible to produce new concepts for an essential understanding of critical phenomena and to create a powerful mathematical apparatus for describing them. The problem mentioned above was considered in a number of books and articles. In most papers devoted to the theory of phase transitions, primary attention is given to determining the universality class of the systems, investigating symmetry properties irrespective of the seed parameters in the initial Hamiltonian, solving recurrence relations (RR), and calculating critical exponents. Universal exponents and combinations of critical amplitudes for thermodynamic characteristics are calculated for spin systems, in particular, for the $3 \mathrm{D}$ Ising model. The problem of dependence of critical amplitudes on the microscopic parameters of the system required a consistent analysis and could be solved successfully together with the main problem in the theory of phase transitions, i.e., the derivation of explicit expressions for thermodynamic characteristics of the system near the phase transition point as functions of temperature and microscopic parameters. Considerable progress in the solution of this problem was made by using the method of collective variables (CV) generalized by Yukhnovskii [1-4] to the case of spin systems. The term collective variables is applied to a special class of variables specific for each individual physical system. The set of CV contains variables associated with order parameters. For this reason, the phase space of CV is most natural for describing a phase transition. For magnetic systems, the $\mathrm{CV} \rho_{\mathbf{k}}$ are the variables associated with modes of spin moment density oscillations, while the order parameter is associated with the variable $\rho_{0}$, in which the subscript " 0 " corresponds to the peak of the Fourier transform of the interaction potential.

This paper supplements a previous study [5] based on the $\mathrm{CV}$ method. In [5], the thermodynamic functions of the classical $n$-vector $3 \mathrm{D}$ magnetic model near $T_{c}$ were calculated in the approximation of the quartic distribution for the spin density oscillation modes ( $\rho^{4}$ model) without taking into account confluent corrections (corrections to scaling). In the present paper, the results of calculating the thermodynamic characteristics of a 3D Ising-like system are obtained using the higher nonGaussian distribution ( $\rho^{6}$ model). In the process of determining these characteristics, a calculation technique for correction-to-scaling terms is elaborated. The employment of the $\rho^{6}$ model for the investigation of the phase transition by the $\mathrm{CV}$ method gives a more precise definition of the calculation results and provides the basis for quantitative analysis of the critical behaviour of $3 \mathrm{D}$ Ising-like systems including the nonuniversal characteristics. On the other hand, the dependence of nonuniversal 


\section{V. PYLYUK}

quantities on the microscopic parameters of the system has not been studied deeply enough so far. Such a study is our aim in this research.

The actual task of the critical phenomena physics at the present time is elaborating the methods giving quantitative description of the critical behaviour of the system without using any adjustable parameters. The approach suggested in the present paper allows us to perform the calculations for a Ising ferromagnet in real $3 \mathrm{D}$ space on the microscopic level without any adjustable parameters. In this case, the new special functions [6-9] appearing in the construction of the phase transition theory using the $\rho^{6}$ model are exploited. We hope that our explicit representations and plots may provide useful benchmarks in studying the dependence of the thermodynamic functions of $3 \mathrm{D}$ Ising-like systems on the parameters of the interaction potential and characteristics of the crystal lattice. The obtained results can be used for interpreting experimental results concerning the behaviour of real materials in the vicinity of the second-order phase transition point, and the computational technique proposed here for thermodynamic characteristics can be used for calculating their thermodynamic functions in the critical region.

\section{CALCULATION SCHEME FOR THE FREE ENERGY OF ISING-LIKE SYSTEM}

We consider a 3D Ising-like system on a simple cubic lattice with period $c$. The Hamiltonian of such a system has the form

$$
H=-\frac{1}{2} \sum_{\mathbf{j}, \mathbf{l}} \Phi(|\mathbf{j}-\mathbf{l}|) \sigma_{\mathbf{j}} \sigma_{\mathbf{l}}
$$

where $\Phi(|\mathbf{j}-\mathbf{l}|)$ is the potential of interaction of particles at sites $\mathbf{j}$ and $\mathbf{l}, \sigma_{\mathbf{j}}$ is the operator of the $z$-component of spin at the $\mathbf{j}$ th site, having two eigenvalues +1 and -1 . The interaction potential is an exponentially decreasing function

$$
\Phi\left(r_{\mathbf{j} \mathbf{l}}\right)=A \exp \left(-\frac{r_{\mathbf{j}} \mathbf{l}}{b}\right)
$$

Here $A$ is a constant, $r_{\mathbf{j}} \mathbf{l}$ is the interparticle distance, and $b$ is the radious of effective interaction. The approximation for the Fourier transform of the interaction potential is taken in the form [3]

$$
\tilde{\Phi}(k)=\left\{\begin{array}{cc}
\tilde{\Phi}(0)\left(1-2 b^{2} k^{2}\right), & k \leq B^{\prime}, \\
0, & B^{\prime}<k \leq B,
\end{array}\right.
$$

where $B$ is the boundary of Brillouin half-zone $(B=$ $\pi / c), B^{\prime}=(b \sqrt{2})^{-1}, \tilde{\Phi}(0)=8 \pi A(b / c)^{3}$.

We shall use here the method of CV [3], which allows us to calculate approximately the expression for partition function and to obtain complete expressions for thermodynamic functions near the phase transition temperature $T_{c}$ in addition to universal quantities (critical exponents).

In the $\mathrm{CV}$ representation for the partition function of the $3 \mathrm{D}$ Ising model, we have

$$
Z=\int \exp \left[\frac{1}{2} \sum_{\mathbf{k}} \beta \tilde{\Phi}(k) \rho_{\mathbf{k}} \rho_{-\mathbf{k}}\right] J(\rho)(d \rho)^{N}
$$

Here the summation over the wave vectors $\mathbf{k}$ is carried out within the first Brillouin zone, $\beta=1 /(k T)$ is the inverse temperature, and the $\mathrm{CV} \rho_{\mathbf{k}}$ are introduced by means of the functional representation for operators of spin density oscillation modes $\hat{\rho}_{\mathbf{k}}=$ $(\sqrt{N})^{-1} \sum \sigma_{\mathbf{l}} \exp (-i \mathbf{k} \mathbf{l})$

$$
\begin{aligned}
& J(\rho)=2^{N} \int \exp \left[2 \pi i \sum_{\mathbf{k}} \omega_{\mathbf{k}} \rho_{\mathbf{k}}+\sum_{n=1}^{\infty}(2 \pi i)^{2 n} N^{1-n}\right. \\
& \left.\times \frac{\mathcal{M}_{2 n}}{(2 n) !} \sum_{\mathbf{k}_{1}, \ldots, \mathbf{k}_{2 n}} \omega_{\mathbf{k}_{1}} \cdots \omega_{\mathbf{k}_{2 n}} \delta_{\mathbf{k}_{1}+\cdots+\mathbf{k}_{2 n}}\right](d \omega)^{N}
\end{aligned}
$$

is the Jacobian of transition from the set of $N$ spin variables $\sigma_{\mathbf{l}}$ to the set of $\mathrm{CV} \rho_{\mathbf{k}}$, and $\delta_{\mathbf{k}_{1}+\ldots+\mathbf{k}_{2 n}}$ is the Kronecker symbol. The variables $\omega_{\mathbf{k}}$ are conjugate to $\rho_{\mathbf{k}}$, and cumulants $\mathcal{M}_{2 n}$ assume constant values (see [3]). The expression for the partition function (4) cannot be calculated exactly due to the presence of an infinitely large number of terms in the exponent (5). For this reason, approximations limiting the number of terms in the exponent of the integrand in (5) are used. A certain approximation of the integrand in the expression for $J(\rho)$ when calculating the explicit form of the Jacobian of the transition determines the choice of the model (models $\rho^{4}, \rho^{6}$, etc.). For $n=1$, we obtain the Gaussian approximation. It leads to classical values of critical exponents. An important condition in describing the critical properties of the Ising model is the use of non-Gaussian densities of measures. The simplest approximation permitting an analysis beyond the classical behaviour corresponds to $n=2$ and is based on quartic density of measure $\left(\rho^{4}\right.$ model). This approximation is used for calculating basic critical exponents of thermodynamic characteristics, complete expressions for these characteristics taking into account confluent corrections, and for analyzing the relation for critical amplitudes (see, for example, [10-12]). In view of an approximate calculation of partition function confined to the $\rho^{4}$ model, the obtained results (critical exponents, amplitudes, and thermodynamic functions) contain a certain dependence on the renormalization group (RG) parameter $s$. This dependence becomes much weaker as the form of the non-Gaussian density of measure becomes more complicated (transition to more complicated models $\rho^{6}(n=3$, see $(5)), \rho^{8}$, and $\left.\rho^{10}\right)$. This is confirmed by an analysis of the behaviour of the criti- 
cal exponents of correlation length $\nu$ for the models $\rho^{2 m}$ $(m=3,4,5)[8,13,14]$ as well as by a direct comparison of the curves describing the temperature dependences of thermodynamic characteristics calculated for the models $\rho^{4}$ and $\rho^{6}$ at different values of the parameter $s[6]$. The dependence of the results of calculations on the parameter $s$ is studied. For each of the $\rho^{2 m}$ models, there exists a preferred value of the parameter $s=s^{*}\left(s^{*}=3.5862\right.$ for the $\rho^{4}$ model, $s^{*}=2.7349$ for the $\rho^{6}$ model, $s^{*}=2.6511$ for the $\rho^{8}$ model, and $s^{*}=2.6108$ for the $\rho^{10}$ model) nullifying the average value of the coefficient in the term with the second power in the effective density of measure at a fixed point. The values of $s$ close to $s^{*}$ are optimal for the given method of calculations. The difference form of RR between the coefficients of effective non-Gaussian densities of measures (expansions for the functions appearing in these relations) operates successfully just in this region of $s$. For such definite methods of division of the phase space of $\mathrm{CV}$ into layers (values of $s$ close to $s^{*}$ ), we obtain reliable results matching with the experimental data and the results of theoretical investigations $[3,6]$. In this paper, the results of calculations based on the $\rho^{6}$ model taking into account the sextic density of measure while integrating the partition function are given for the values of $s$ equaling 2, 2.7349, and 3 (i.e., optimal values for the given method of calculations).

In our earlier publication [15], we proposed a new method of calculation of the equation of state for a $3 \mathrm{D}$ Ising system on microscopic level in the approximation of the above-mentioned $\rho^{6}$ model. This equation attracts the attention of many scientists (see, for example, [16] in which the equation of state for the systems of universality class of the $3 \mathrm{D}$ Ising model is analyzed by using the field-theory approach with RG technique). The cor- rectness of the choice of the $\rho^{6}$ model for investigations is confirmed in $[17,18]$, where the effective potential is studied for the scalar field theory in three dimensions in the symmetric and spontaneously broken phases, respectively. In this case, probability distributions of average magnetization in the $3 \mathrm{D}$ Ising model in an external field obtained with the help of the Monte Carlo method were used. Tsypin $[17,18]$ proved that the term with the sixth power of the variable in the effective potential plays an important role. In [17], the values of universal four- and six-point coupling constants were calculated. Dimensionless six- and eight-point effective coupling constants were calculated by Sokolov et al. [19] in the three-loop approximation using the field-theory RG approach. An estimate for the dimensionless sextic effective coupling constant in the case of the 3D Ising model in the four-loop approximation is given in [20].

In this paper, the $\rho^{6}$ model is used for developing the method of calculation of expressions for thermodynamic functions of the $3 \mathrm{D}$ Ising system taking into account the terms determining the correction to scaling. The calculations are made above and below the phase transition temperature $T_{c}$ (high- and low-temperature regions). The obtained expressions for basic critical amplitudes and the amplitudes of the first confluent correction make it possible to analyze their dependence on microscopic parameters of the system (the range $b$ of potential and the lattice constant $c$ ).

We shall proceed from the expression for partition function in the approximation of the $\rho^{6}$ model. Putting $n=3$ in (5) and carrying out integration in (4) with respect to the variables $\rho_{\mathbf{k}}$ and $\omega_{\mathbf{k}}$ with indices $B^{\prime}<k \leq B$, followed by the integration with respect to $N^{\prime}$ variables $\omega_{\mathbf{k}}$, we obtain

$Z=2^{N} 2^{\left(N^{\prime}-1\right) / 2} e^{a_{0}^{\prime} N^{\prime}} \int \exp \left[-\frac{1}{2} \sum_{k \leq B^{\prime}} d^{\prime}(k) \rho_{\mathbf{k}} \rho_{-\mathbf{k}}-\sum_{l=2}^{3} \frac{a_{2 l}^{\prime}}{(2 l) !\left(N^{\prime}\right)^{l-1}} \sum_{k_{1}, \ldots, k_{2 l} \leq B^{\prime}} \rho_{\mathbf{k}_{1}} \cdots \rho_{\mathbf{k}_{2 l}} \delta_{\mathbf{k}_{1}+\cdots+\mathbf{k}_{2 l}}\right](d \rho)^{N^{\prime}}$

Here

$$
N^{\prime}=N s_{0}^{-3}, \quad s_{0}=B / B^{\prime}=\pi \sqrt{2} b / c, \quad d^{\prime}(k)=a_{2}^{\prime}-\beta \tilde{\Phi}(k) .
$$

The coefficients $a_{2 l}^{\prime}$ are defined as

$$
\begin{aligned}
& a_{0}^{\prime}=\ln Q(\mathcal{M}), \quad Q(\mathcal{M})=\left(12 s_{0}^{3}\right)^{1 / 4} \pi^{-1} I_{0}\left(\eta^{\prime}, \xi^{\prime}\right), \\
& a_{2}^{\prime}=\left(12 s_{0}^{3}\right)^{1 / 2} F_{2}\left(\eta^{\prime}, \xi^{\prime}\right), \quad a_{4}^{\prime}=12 s_{0}^{3} C\left(\eta^{\prime}, \xi^{\prime}\right), \quad a_{6}^{\prime}=\left(12 s_{0}^{3}\right)^{3 / 2} N\left(\eta^{\prime}, \xi^{\prime}\right)
\end{aligned}
$$

and are functions of $s_{0}$, i.e., of the ratio $b / c$ (see Table 1 ). In this expressions, the role of the arguments is played by the quantities

$$
\eta^{\prime}=\sqrt{3} s_{0}^{3 / 2}, \quad \xi^{\prime}=\frac{8 \sqrt{3}}{15 s_{0}^{3 / 2}}
$$




\section{V. PYLYUK}

The special function $C\left(\eta^{\prime}, \xi^{\prime}\right)$ and $N\left(\eta^{\prime}, \xi^{\prime}\right)$ have the form

$$
C\left(\eta^{\prime}, \xi^{\prime}\right)=-F_{4}\left(\eta^{\prime}, \xi^{\prime}\right)+3 F_{2}^{2}\left(\eta^{\prime}, \xi^{\prime}\right), \quad N\left(\eta^{\prime}, \xi^{\prime}\right)=F_{6}\left(\eta^{\prime}, \xi^{\prime}\right)-15 F_{4}\left(\eta^{\prime}, \xi^{\prime}\right) F_{2}\left(\eta^{\prime}, \xi^{\prime}\right)+30 F_{2}^{3}\left(\eta^{\prime}, \xi^{\prime}\right)
$$

where

$$
F_{2 l}\left(\eta^{\prime}, \xi^{\prime}\right)=I_{2 l}\left(\eta^{\prime}, \xi^{\prime}\right) / I_{0}\left(\eta^{\prime}, \xi^{\prime}\right), \quad I_{2 l}\left(\eta^{\prime}, \xi^{\prime}\right)=\int_{0}^{\infty} t^{2 l} \exp \left(-\eta^{\prime} t^{2}-t^{4}-\xi^{\prime} t^{6}\right) d t
$$

\begin{tabular}{lccccccc}
\hline$b$ & $b_{\mathrm{I}}$ & \multicolumn{1}{c}{$b_{\mathrm{II}}$} & \multicolumn{1}{c}{$b_{\mathrm{III}}$} & $c$ & $2 c$ & $5 c$ & $7 c$ \\
\hline$a_{0}^{\prime}$ & -1.0196 & -0.9863 & -0.9764 & -0.9218 & -0.9193 & -0.9190 & -0.9189 \\
$a_{2}^{\prime}$ & 0.7023 & 0.7820 & 0.8083 & 0.9887 & 0.9986 & 0.9999 & 1.0000 \\
$a_{4}^{\prime}$ & 0.2212 & 0.2163 & 0.2086 & 0.0220 & 0.0028 & 0.0002 & 0.0000 \\
$a_{6}^{\prime}$ & 0.4379 & 0.3895 & 0.3547 & 0.0031 & 0.0000 & 0.0000 & 0.0000 \\
\hline
\end{tabular}

Table 1. Values of coefficients $a_{2 l}^{\prime}$ for various $b$.

It can be seen from Table 1 that $a_{2}^{\prime} \rightarrow 1$ for $b \gg c$, $a_{2 l}^{\prime} \rightarrow 0$ for $l \geq 2$, and the integrand in formula (6) has the form close to the Gaussian distribution. In the case when the range of the potential and the lattice constant are commensurate, the coefficients $a_{2 l}^{\prime}$ differ from zero for all values of $l \geq 0$, and we must take into account in the exponent of the integrand in (6) the terms proportional to higher degrees of the variable $\rho_{\mathbf{k}}$ in addition to the Gaussian term. Henceforth, we shall consider just this case. The value of $b=b_{\mathrm{I}}=c /(2 \sqrt{3})$ corresponds to the interaction between nearest neighbours, $b=b_{\mathrm{II}}=0.3379 c$ to the interaction between the nearest and next-nearest neighbours, and $b=b_{\mathrm{III}}=0.3584 c$ to the nearest, next-nearest, and third neighbours [21]. At these values of $b$ and small values of the wave vectors $\mathbf{k}$, the parabolic approximation of the Fourier transform of the exponentially decreasing interaction potential corresponds to the analogous approximation of the Fourier transform for the interaction potentials of the above-mentioned neighbours.

The increase in the number of terms in expressions (5) and (6) corresponds to a complication of the form of the non-Gaussian density of measure (complication of the model). Critical phenomena in a 3D Ising ferromagnet within the $\mathrm{CV}$ method can be described quantitatively even in the $\rho^{6}$ model approximation. The confinement to the quartic approximation in formulas (5) and (6) allows us to go beyond the classical analysis and to describe all qualitative aspects of the second-order phase transition, while the sextic approximation ensures a more adequate quantitative description of the critical properties of a spin system. This is confirmed by calculation as well as an analysis of the behaviour of the coefficients in the initial expression for partition function and the critical exponent of the correlation length $\nu$ for the sequence of $\rho^{4}, \rho^{6}, \rho^{8}$, and $\rho^{10}$ models $[3,8,13,14]$ as well as by the calculation and comparison of thermodynamic functions for the models $\rho^{4}$ and $\rho^{6}[6]$ and by comparison of the results of our calculations with other available data (see, for example, $[6,7]$ ). The analysis of the abovementioned sequence of the model was necessary for estimating the convergence of the procedure for calculating the critical exponent $\nu$, for finding its dependence on the RG parameter $s$, and for establishing whether it is expedient to use higher densities of measures. It was found that as the form of the density of measure becomes more complicated, the dependence of the critical exponent $\nu$ on the RG parameter $s$ becomes weaker gradually, and starting from the sextic density of measure, the value of the exponent $\nu$, having a tendency to saturation with increasing $m$ (which characterizes the order of the $\rho^{2 m}$ model or determines the summation limit in formula (5), $m=2,3,4,5)$ changes insignificantly. It is also interesting that for $m \geq 3$, the numerical values of the coefficients $a_{2 l}^{\prime}$ appearing in the partition function (relations similar to (8) and (6)) vary insignificantly upon an increase in the order of the density of measure, i. e., upon an increase in the number of terms in (5).

An advantage of the $\mathrm{CV}$ method in the description of phase transitions is the presence of a variable associated with the order parameter among the $\mathrm{CV} \rho_{\mathbf{k}}$. Such a variable for the Ising model is $\rho_{0}$. We cannot single out the contribution from $\rho_{0}$ alone in expression (6) since all the variables $\rho_{\mathbf{k}}$ are interconnected. We shall use the method of "layer-by-layer" integration of (6) with respect to variables $\rho_{\mathbf{k}}$ proposed by Yukhnovskii [3]. The integration begins from the variables $\rho_{\mathbf{k}}$ with a large value of $k$ (of the order of the Brillouin half-zone boundary) and termi- 
nates at $\rho_{\mathbf{k}}$ with $k \rightarrow 0$. For this purpose, we divide the phase space of the $\mathrm{CV} \rho_{\mathbf{k}}$ into layers with the division parameter $s$. In each $n$th layer (corresponding to the region of wave vectors $B_{n+1}<k \leq B_{n}, B_{n+1}=B_{n} / s, s>1$ ), the Fourier transform of the potential $\tilde{\Phi}(k)$ is replaced by its average value (arithmetic mean in the given case). To simplify the presentation, we assume that the correction for the potential averaging is zero, although it can be taken into account if necessary [3]. Including this correction leads to a nonzero value of the critical exponent $\eta$ characterizing the behaviour of the pair correlation function for $T=T_{c}$. As a result of step-by-step calculation of partition function, the number of integration variables in the expression for this quantity decreases gradually. After the integration over $n+1$ layers of the CV space, we obtain

$$
Z=2^{N} 2^{\left(N_{n+1}-1\right) / 2} Q_{0} Q_{1} \cdots Q_{n}\left[Q\left(P_{n}\right)\right]^{N_{n+1}} \int \mathcal{W}_{6}^{(n+1)}(\rho)(d \rho)^{N_{n+1}}
$$

Here $N_{n+1}=N^{\prime} s^{-3(n+1)}$, and

$$
\begin{aligned}
& Q_{0}=\left[e^{a_{0}^{\prime}} Q(d)\right]^{N^{\prime}}, \quad Q_{1}=\left[Q(P) Q\left(d_{1}\right)\right]^{N_{1}}, \ldots, \\
& Q_{n}=\left[Q\left(P_{n-1}\right) Q\left(d_{n}\right)\right]^{N_{n}}, \quad Q\left(d_{n}\right)=2\left(24 / a_{4}^{(n)}\right)^{1 / 4} I_{0}\left(h_{n}, \alpha_{n}\right), \\
& Q\left(P_{n}\right)=\pi^{-1}\left(s^{3} a_{4}^{(n)} / C\left(h_{n}, \alpha_{n}\right)\right)^{1 / 4} I_{0}\left(\eta_{n}, \xi_{n}\right) .
\end{aligned}
$$

The basic arguments

$$
h_{n}=d_{n}\left(B_{n+1}, B_{n}\right)\left(6 / a_{4}^{(n)}\right)^{1 / 2}, \quad \alpha_{n}=\frac{\sqrt{6}}{15} a_{6}^{(n)} /\left(a_{4}^{(n)}\right)^{3 / 2}
$$

are determined by the mean value of the coefficient $d_{n}(k)$ in the $n$th layer of the phase space of $\mathrm{CV}$, i.e., by $d_{n}\left(B_{n+1}, B_{n}\right)$ as well as the quantities $a_{4}^{(n)}$ and $a_{6}^{(n)}$. The effective sextic density of measure of the $(n+1)$ th block structure $\mathcal{W}_{6}^{(n+1)}(\rho)$ has the form

$$
\mathcal{W}_{6}^{(n+1)}(\rho)=\exp \left[-\frac{1}{2} \sum_{k \leq B_{n+1}} d_{n+1}(k) \rho_{\mathbf{k}} \rho_{-\mathbf{k}}-\sum_{l=2}^{3} \frac{a_{2 l}^{(n+1)}}{(2 l) ! N_{n+1}^{l-1}} \sum_{k_{1}, \ldots, k_{2 l} \leq B_{n+1}} \rho_{\mathbf{k}_{1}} \cdots \rho_{\mathbf{k}_{2 l}} \delta_{\mathbf{k}_{1}+\cdots+\mathbf{k}_{2 l}}\right] \text {. }
$$

Here $B_{n+1}=B^{\prime} s^{-(n+1)}, d_{n+1}(k)=a_{2}^{(n+1)}-\beta \tilde{\Phi}(k), a_{2 l}^{(n+1)}$ are renormalized values of the coefficients $a_{2 l}^{\prime}$ after integration over $n+1$ layers of the phase space of CV. The intermediate variables $\eta_{n}, \xi_{n}$ are functions of $h_{n}$ and $\alpha_{n}$ and are defined as

$$
\eta_{n}=\left(6 s^{3}\right)^{1 / 2} F_{2}\left(h_{n}, \alpha_{n}\right)\left[C\left(h_{n}, \alpha_{n}\right)\right]^{-1 / 2}, \quad \xi_{n}=\frac{\sqrt{6}}{15} s^{-3 / 2} N\left(h_{n}, \alpha_{n}\right)\left[C\left(h_{n}, \alpha_{n}\right)\right]^{-3 / 2},
$$

where the form of the special functions $C\left(h_{n}, \alpha_{n}\right)$ and $N\left(h_{n}, \alpha_{n}\right)$ is given by (10). The coefficients $d_{n}\left(B_{n+1}, B_{n}\right), a_{4}^{(n)}$, and $a_{6}^{(n)}$ are connected with the coefficients for the $(n+1)$ th layer through the RR $[8,22]$ whose solutions $[15,22]$ are used for calculating the free energy of the system.

The basic idea of the calculation of explicit expressions for free energy and other thermodynamic functions of the system near $T_{c}$ on microscopic level $\left(\tau<\tau^{*} \sim\right.$ $\left.10^{-2}, \tau=\left(T-T_{c}\right) / T_{c}\right)$ lies in the separate inclusion of contributions from short-wave and long-wave modes of spin moment density oscillations $[3,10,23]$.

Short-wave modes are characterized by a RG symmetry and are described by a non-Gaussian density of measure. They correspond to the region of critical regime (CR) observed above as well as below $T_{c}$. In this case, the RG method is used (see, for example, [24]). The calculation of the expression describing the contribution from 


\section{V. PYLYUK}

short-wave modes of spin moment density oscillations to free energy involves the summation of partial free energies over the layers of the phase space of CV up to the point, at which the system leaves the $\mathrm{CR}$ region. In this case, it is important to obtain an explicit dependence on the number of the layer. For this purpose, the solutions of RR are used. Taking into account the larger eigenvalue $\left(E_{1}>1\right)$ of the $\mathrm{RG}$ linear transformation matrix, we can describe the main singularity for specific heat near $T_{c}$. Smaller eigenvalues $\left(E_{2}<1\right.$ and $\left.E_{3}<1\right)$ are responsible for the emergence of corrections to scaling. The inclusion of short-wave modes of spin density oscillations leads to a renormalization of the dispersion of the distribution describing long-wave modes. The region of the limiting Gaussian regime (LGR) for $T>T_{c}$ or the region of the inverse Gaussian regime (IGR) for $T<T_{c}$ is associated with these long-wave modes. The fluctuations in the LGR region are characterized by the Gaussian distribution. In contrast to the LGR, the IGR is described by a non-Gaussian measure density. The distribution acquires the Gaussian form only as a result of separating the free energy of the ordering. The way, in which the contribution from long-wave modes of oscillations to free energy of the system is taken into account, differs qualitatively from the method of calculating the short-wave part of partition function. The calculation of this contribution is based on the use of the Gaussian density of measure as the basis density. We have developed a direct method of calculations with the results obtained by taking into account the short-wave modes as initial parameters.

It should be noted that our calculations are valid in a small neighbourhood of $T_{c}$. The solutions of RR make it possible to calculate the size of this critical region. Indeed, using these solutions and the condition for the existence of $\mathrm{CR}$ (the exit from this regime for $n \rightarrow 1$, described by terms proportional to $E_{1}^{n}$, does not prevail over the entry to this regime, which is described by terms proportional to $E_{2}^{n}$ and $E_{3}^{n}$ ), we can determine the temperature range $\tau<\tau^{*}$, in which the CR corresponding to the presence of strongly correlated spin blocks occurs. The value of $\tau^{*}$ is equal to the magnitude of the smallest root of the three equations obtained on the basis of solutions of RR. The value of $\tau^{*}$ determined in this way is of the order of a few hundredths for commensurate $b$ and $c$ [25]. For large values of $b$, it is important to take correctly into account the entry to the CR. In this case, the value of $\tau^{*}$ can be obtained by imposing the condition that the entry to CR prevails over the exit from this regime for $n \rightarrow n_{0}$, where the layer number $n_{0}$ as a function of $b$ can be determined from RR [8,22], for example, proceeding from the relation for $a_{4}^{(n)}$ (see Table 2). The data contained in Table 2 show that the critical region is practically absent for large $b$. This is not surprising since the condition $b \gg c$ corresponds to the transition to the model with long-range interaction, which is based on the Gaussian distribution of spin density oscillation modes (see (6) and Table 1) and demonstrates the classical critical behaviour.

A distinctive feature of RR solutions is a specific dependence of one of their coefficients on temperature.
The expression for this coefficient can be used to derive an equation for the quantity $\beta_{c} \tilde{\Phi}(0)$ determining the phase transition temperature (see [22]). A direct analytic method for calculating the phase transition temperature in the $\rho^{4}$ model approximation is developed in $[21,26]$.

\begin{tabular}{cccc}
\hline$b$ & $4 c$ & $5 c$ & $7 c$ \\
\hline$n_{0}$ & 7 & 8 & 9 \\
$\tau^{*}$ & $0.8266 \times 10^{-8}$ & $0.6274 \times 10^{-9}$ & $0.4680 \times 10^{-10}$ \\
\hline
\end{tabular}

Table 2. Values of $n_{0}$ and $\tau^{*}$ for large values of the interaction potential range $b$ and the RG parameter $s=3$.

The calculations for a 3D Ising-like system in these papers are illustrated by the consideration of the simple cubic lattice case. The influence of the short-wave part of the Fourier transform of the exponentially decreasing interaction potential $(\tilde{\Phi}(k)$ is the small constant at $B^{\prime}<k<B$ ) on $T_{c}$ is studied. Our numerical value $\beta_{c} J=$ $0.211\left(\rho^{4}\right.$ model) [21] for the case when the potential parameters correspond to the nearest-neighbour interaction with the constant $J$ accords with the conclusions of other authors, for example, with the value $\left(\beta_{c} J\right)^{-1}=4.5103$ (or $\beta_{c} J=0.2217$ ) $[27,28]$ or $\beta_{c} J=0.221654(1)$ [29] calculated using the high-temperature series data. Similar values are also obtained using the real space RG method based on the cumulant expansion $\left(\beta_{c} J=0.22401\right.$ [30]) and the Monte Carlo method $\left(\beta_{c} J=0.221654 \pm 0.000006\right.$ $\left.[31], \beta_{c} J=0.2216595 \pm 0.0000026[32-34]\right)$. Liu and Fisher [35] preferred $\beta_{c} J=0.221692, \beta_{c} J=0.221630$, and $\beta_{c} J=0.221620$ for the simple cubic Ising lattice and the three selected values of the susceptibility exponent $\gamma(\gamma=1.250, \gamma=1.2395$, and $\gamma=1.237)$, respectively. The dependence of $\beta_{c} A$ ( $A$ is the constant appearing in the interaction potential (2)) on the ratio of the potential range $b$ to the lattice constant $c$ is shown in Fig. 1 for the $\rho^{6}$ model. Here and below, the curves are plotted for the RG parameters $s=3$.

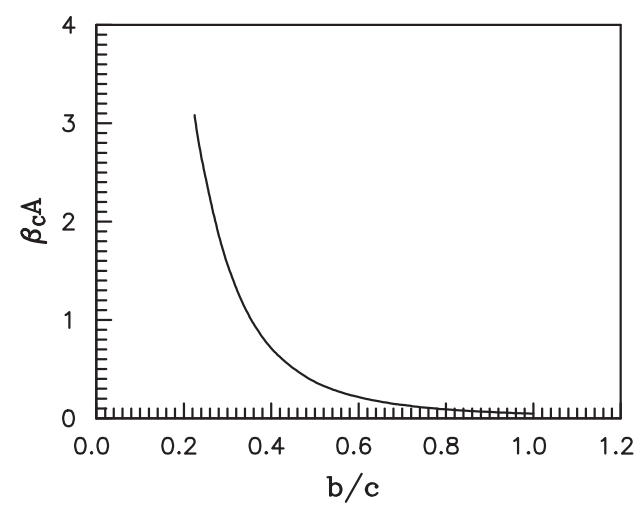

Fig. 1. Dependence of the inverse phase transition temperature on the ratio of the interaction potential range to the lattice constant. 
Calculating separately the contributions to free energy from short- and long-wave modes of spin density oscillations $\left(F_{\mathrm{CR}}^{+}\right.$and $F_{\mathrm{LGR}}$ at $T>T_{c}$ or $F_{\mathrm{CR}}^{-}$and $F_{\mathrm{IGR}}$ at $T<T_{c}$ ), we can obtain the complete expression for the free energy of the system:

$$
F= \begin{cases}F_{0}+F_{\mathrm{CR}}^{+}+F_{\mathrm{LGR}}, & T>T_{c}, \\ F_{0}+F_{\mathrm{CR}}^{-}+F_{\mathrm{IGR}}, & T<T_{c} .\end{cases}
$$

Here $F_{0}=-k T N \ln 2$ is the free energy of $N$ noninteracting spins. Detailed calculations of the contributions to free energy of the system from short- and long-wave modes and the coefficients of complete expressions for thermodynamic characteristics are presented in [36,37]. The paper [36] was devoted to the critical behaviour of a $3 \mathrm{D}$ Ising-like system in the high-temperature region.
The low-temperature region was considered in [37]. The calculations are performed on the basis of the $\rho^{6}$ model taking into account the first confluent correction (which is determined by the term proportional to $\tau^{\Delta_{1}}\left(T>T_{c}\right)$ or $\left.|\tau|^{\Delta_{1}}\left(T<T_{c}\right), \Delta_{1}=-\ln E_{2} / \ln E_{1}\right)$. We disregard the second confluent correction (which is determined by the term proportional to $\tau^{\Delta_{2}}\left(T>T_{c}\right)$ or $|\tau|^{\Delta_{2}}\left(T<T_{c}\right)$, $\Delta_{2}=-\ln E_{3} / \ln E_{1}$ ). This is due to the fact that the contribution from the first confluent correction to thermodynamic functions of the model near $T_{c}$ is more significant than the small contribution from the second correction $\left(|\tau| \ll 1, \Delta_{1}\right.$ is of the order of 0.5 , and $\Delta_{2}>2$, see [6]). Below, the final expressions for thermodynamic characteristics of the system are given in the case of a zero external field. The curves of the dependences of these characteristics on temperature and microscopic parameters are plotted using the calculation results in the highand low-temperature regions.

\section{THERMODYNAMIC CHARACTERISTICS NEAR $T_{C}$ AS FUNCTIONS OF TEMPERATURE AND MICROSCOPIC PARAMETERS OF THE SYSTEM}

In accordance with (17), the total free energy of the system assumes the form

$$
F= \begin{cases}-k T N^{\prime}\left[\gamma_{0}+\gamma_{1} \tau+\gamma_{2} \tau^{2}+\gamma_{3}^{(0)+} \tau^{3 \nu}+\gamma_{3}^{(1)+} \tau^{3 \nu+\Delta_{1}}\right], & T>T_{c} \\ -k T N^{\prime}\left[\gamma_{0}-\gamma_{1}|\tau|+\gamma_{2}|\tau|^{2}+\gamma_{3}^{(0)-}|\tau|^{3 \nu}+\gamma_{3}^{(1)-}|\tau|^{3 \nu+\Delta_{1}}\right], & T<T_{c}\end{cases}
$$

where $\nu=\ln s / \ln E_{1}$ is the critical exponent of the correlation length. The terms proportional to integral powers of $\tau$ in (18) appear exclusively due to inclusion of short-wave modes of oscillations. The terms proportional to $\tau^{3 \nu}$ and $\tau^{3 \nu+\Delta_{1}}$ (nonanalytic component of free energy) are formed as a result of inclusion of short-wave as well as long-wave modes of oscillations. The first confluent correction appears due to the smaller eigenvalue $E_{2}$ of the RG linear transformation matrix taken into account in the solutions of RR. All the coefficients in expression for $F$ (see $[36,37])$ are functions of microscopic parameters of the system, i.e., the effective radius $b$ of the potential, the Fourier transform $\tilde{\Phi}(0)$ of the potential for $k=0$, and the lattice constant $c$. The values of the coefficients $\gamma_{0}, \gamma_{1}$, and $\gamma_{2}$ are independent of whether calculations are made for a temperature above or below the phase transition point. The coefficients $\gamma_{3}^{(l) \pm}(l=0,1)$ can be written as a product of the quantity $\bar{\gamma}_{3}^{(l) \pm}$, which is universal relative to microscopic parameters, and the nonuniversal factor $c_{\nu}^{3} c_{\Delta_{1}}^{l}[36,38]$, which is a function of these parameters:

$$
\gamma_{3}^{(l) \pm}=c_{\nu}^{3} c_{\Delta_{1}}^{l} \bar{\gamma}_{3}^{(l) \pm}, \quad l=0,1
$$

The "+" and "-" signs refer to temperatures above and below $T_{c}$, respectively. Numerical values of the coefficients $\bar{\gamma}_{3}^{(l) \pm}$ are given in $[36,37]$.

The main advantage of the expression for $F$ is the presence of relations connecting its coefficients with microscopic parameters of the system and the coordinates of a fixed point of RR. Leading critical amplitudes and the amplitudes of the confluent correction for the specific heat and other thermodynamic characteristics of the system can be presented in a similar way.

The coefficients of entropy, internal energy, and specific heat can be expressed in terms of the coefficients of free energy. Taking into account the first confluent correction, we obtain the following expressions for entropy $S$, internal energy $U$, and specific heat $C$ :

$$
S= \begin{cases}k N^{\prime}\left[s^{(0)}+c_{0} \tau+u_{3}^{(0)+} \tau^{1-\alpha}+u_{3}^{(1)+} \tau^{1-\alpha+\Delta_{1}}\right], & T>T_{c} \\ k N^{\prime}\left[s^{(0)}-c_{0}|\tau|-u_{3}^{(0)-}|\tau|^{1-\alpha}-u_{3}^{(1)-}|\tau|^{1-\alpha+\Delta_{1}}\right], & T<T_{c}\end{cases}
$$




\section{V. PYLYUK}

$$
\begin{gathered}
U= \begin{cases}k T N^{\prime}\left[\gamma_{1}+u_{1} \tau+u_{3}^{(0)+} \tau^{1-\alpha}+u_{3}^{(1)+} \tau^{1-\alpha+\Delta_{1}}\right], & T>T_{c}, \\
k T N^{\prime}\left[\gamma_{1}-u_{1}|\tau|-u_{3}^{(0)-}|\tau|^{1-\alpha}-u_{3}^{(1)-}|\tau|^{1-\alpha+\Delta_{1}}\right], & T<T_{c},\end{cases} \\
C= \begin{cases}k N^{\prime}\left[c_{0}+c_{3}^{(0)+} \tau^{-\alpha}+c_{3}^{(1)+} \tau^{\Delta_{1}-\alpha}\right], & T>T_{c}, \\
k N^{\prime}\left[c_{0}+c_{3}^{(0)-}|\tau|^{-\alpha}+c_{3}^{(1)-}|\tau|^{\Delta_{1}-\alpha}\right], & T<T_{c} .\end{cases}
\end{gathered}
$$

Here

$$
\begin{aligned}
& s^{(0)}=\gamma_{0}+\gamma_{1}, \quad c_{0}=2\left(\gamma_{1}+\gamma_{2}\right), \quad u_{1}=\gamma_{1}+2 \gamma_{2}, \\
& u_{3}^{(l) \pm}=c_{\nu}^{3} c_{\Delta_{1}}^{l} \bar{u}_{3}^{(l) \pm}, \quad l=0,1, \\
& \bar{u}_{3}^{(0) \pm}=3 \nu \bar{\gamma}_{3}^{(0) \pm}, \quad \bar{u}_{3}^{(1) \pm}=\left(3 \nu+\Delta_{1}\right) \bar{\gamma}_{3}^{(1) \pm}, \\
& c_{3}^{(l) \pm}=c_{\nu}^{3} c_{\Delta_{1}}^{l} \bar{c}_{3}^{(l) \pm}, \quad \bar{c}_{3}^{(0) \pm}=3 \nu(3 \nu-1) \bar{\gamma}_{3}^{(0) \pm}, \\
& \bar{c}_{3}^{(1) \pm}=\left(3 \nu+\Delta_{1}\right)\left(3 \nu+\Delta_{1}-1\right) \bar{\gamma}_{3}^{(1) \pm},
\end{aligned}
$$

and $\alpha=2-3 \nu$ is the critical exponent of the specific heat.

The formula for specific heat (see (20)) can be rewritten in the form $[39,40]$

$$
\begin{gathered}
\frac{C}{k N^{\prime}}=\frac{A^{ \pm}}{\alpha}|\tau|^{-\alpha}\left(1+\alpha a_{c}^{ \pm}|\tau|^{\Delta_{1}}\right)+B^{ \pm}, \\
A^{ \pm}=c_{\nu}^{3} \alpha \bar{c}_{3}^{(0) \pm}, \quad a_{c}^{ \pm}=\frac{c_{\Delta_{1}}}{\alpha} \frac{\bar{c}_{3}^{(1) \pm}}{\bar{c}_{3}^{(0) \pm}}, \quad B^{ \pm}=c_{0} .
\end{gathered}
$$

The proposed method makes it possible to calculate such important characteristics of the system as the average spin moment (which plays the role of the order parameter for the system under investigation)

$$
\langle\sigma\rangle=\langle\sigma\rangle^{(0)}|\tau|^{\beta}\left(1+\langle\sigma\rangle^{(1)}|\tau|^{\Delta_{1}}\right)
$$

and the susceptibility per particle

$$
\chi=\Gamma^{ \pm}|\tau|^{-\gamma}\left(1+a_{\chi}^{ \pm}|\tau|^{\Delta_{1}}\right) \frac{\mu_{B}^{2}}{\tilde{\Phi}(0)} .
$$

Here $\mu_{B}$ is the Bohr magneton, $\beta=\nu / 2$ and $\gamma=2 \nu$ are the critical exponents of the average spin moment and the susceptibility, respectively. The leading critical amplitudes and the amplitudes of the first confluent correction for the average spin moment and the susceptibility are obtained in $[36,37]$ for various values of the interaction potential range.

Using the explicit expressions presented here, we can investigate the dependences of thermodynamic characteristics of a 3D Ising-like system on its microscopic pa- rameters. The temperature-dependence curves for the average spin moment $\langle\sigma\rangle$, entropy $S / k N$, specific heat $C / k N$, and the susceptibility $\chi$ (in the units of $\mu_{B}^{2} / A$, $A=\tilde{\Phi}(0) /\left[8 \pi(b / c)^{3}\right]$ is the interaction potential constant) near $T_{c}$ for different values of the effective radius $b$ of the potential are shown in Figs. 2, 3, 4, and 5 .

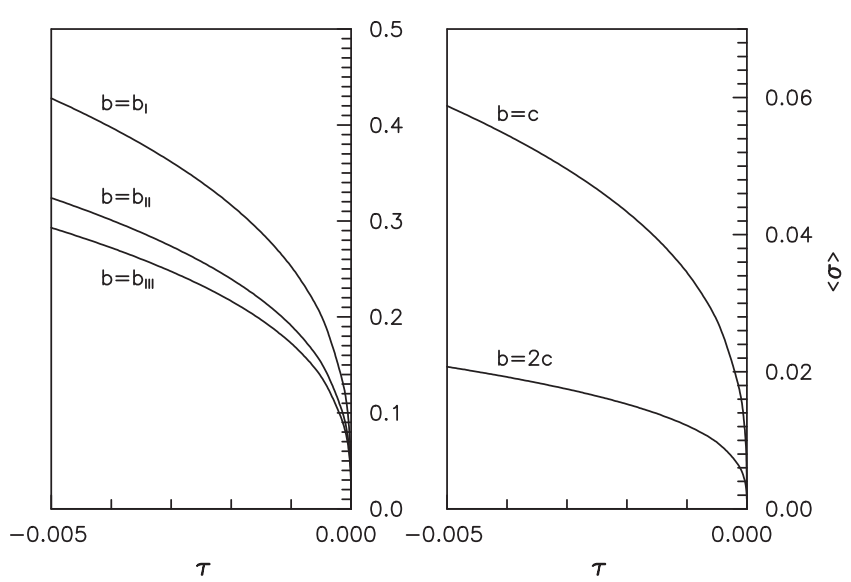

Fig. 2. Temperature dependence of average spin moment of the system in the $\rho^{6}$ model approximation for various values of the effective radius $b$ of the potential: $b_{\mathrm{I}}=c /(2 \sqrt{3})$; $b_{\mathrm{II}}=0.3379 c ; b_{\mathrm{III}}=0.3584 c ; c$ and $2 c$.
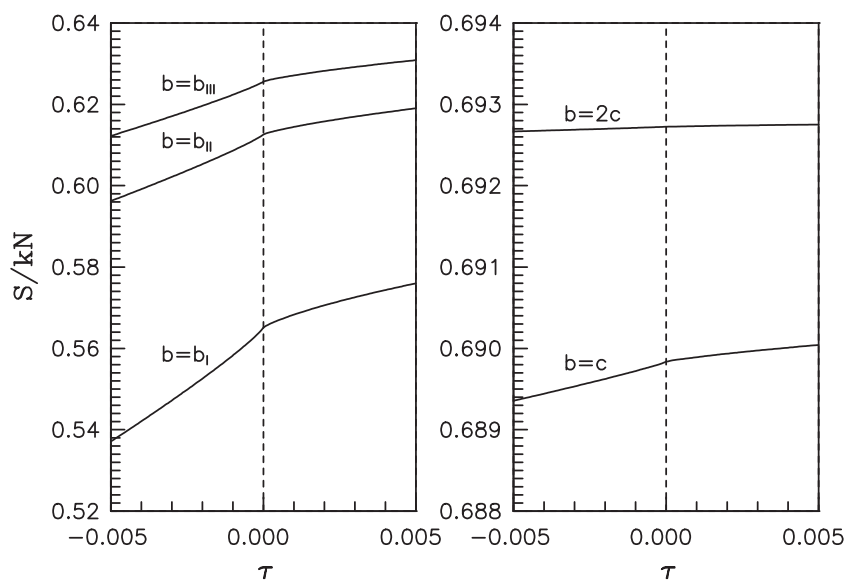

Fig. 3. Dependence of the entropy of the system on $\tau$. Notation is the same as in Fig. 2. 


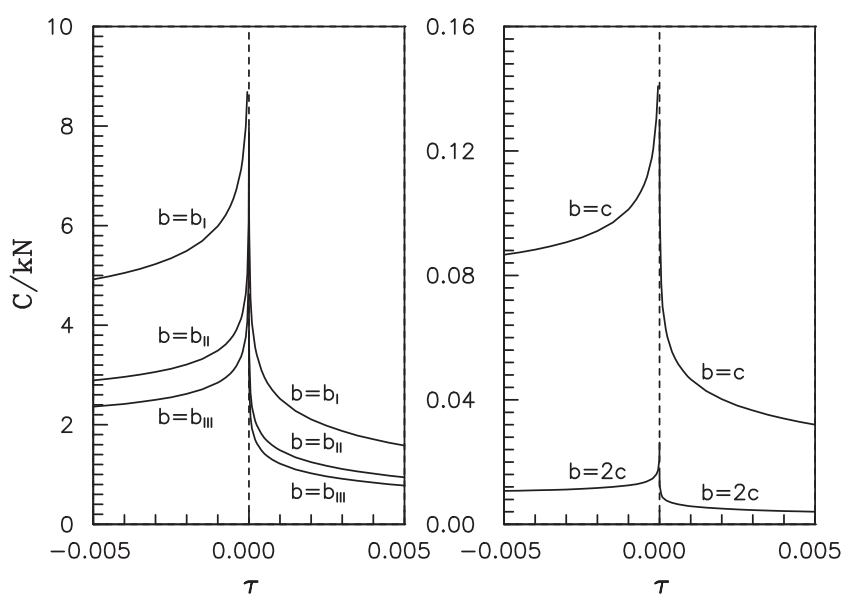

Fig. 4. Specific heat of the spin system for various values of $b$. Notation is the same as in Fig. 2.
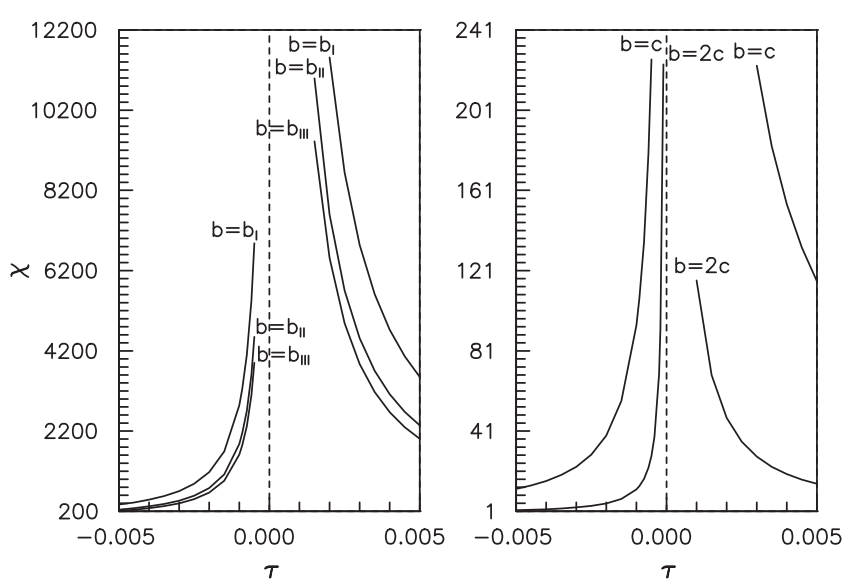

Fig. 5. Temperature dependence of the susceptibility of the system for various values of $b$. Notation is the same as in Fig. 2.

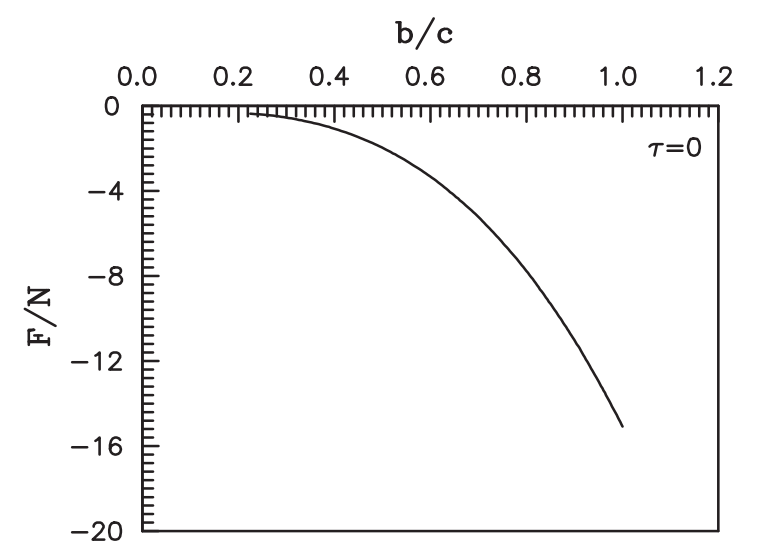

Fig. 6. Dependence of the free energy of the system at the phase transition point $(\tau=0)$ on the ratio of the effective radius $b$ of exponentially decreasing interaction potential to the simple cubic lattice constant $c$.
The evolution of the free energy $F / N$ of the system (in the units of $A$ ) at the phase transition point $(\tau=0)$ as well as of the average spin moment $\langle\sigma\rangle$ for $\tau=-10^{-3}$ and the specific heat $C / k N$ of the system for $|\tau|=10^{-3}$ with increasing ratio of the effective radius $b$ of the potential to the lattice constant $c$ is plotted in Figs. 6, 7, and 8 .

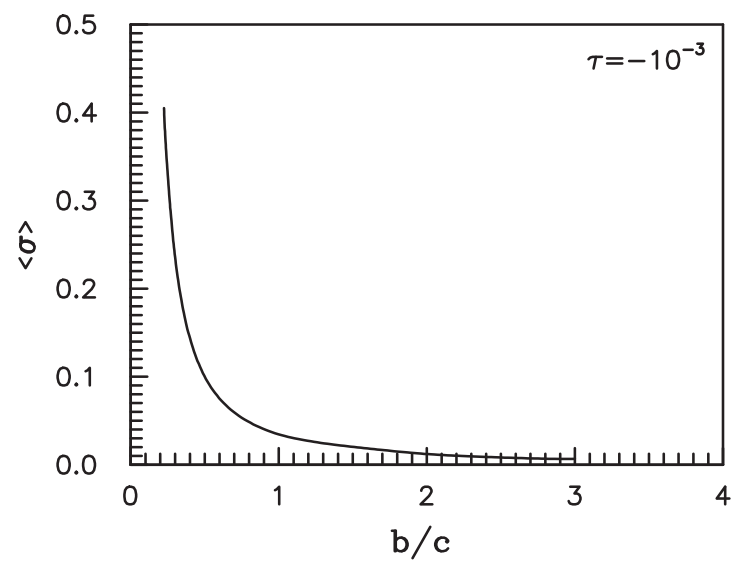

Fig. 7. Behaviour of the average spin moment for $\tau=-10^{-3}$ with increasing ratio $b / c$.

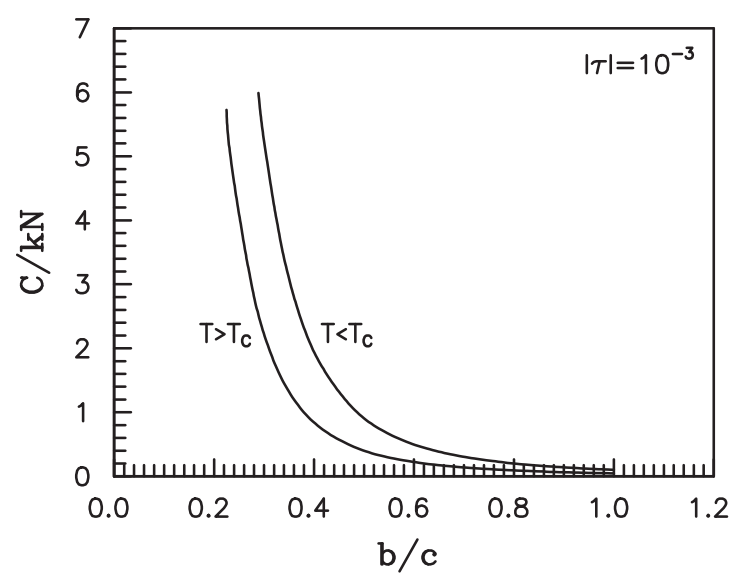

Fig. 8. Evolution of the specific heat of the system for $|\tau|=10^{-3}$ with increasing ratio $b / c$.

\section{CONCLUSIONS}

The analytic method for calculating the thermodynamic functions of $3 \mathrm{D}$ Ising-like systems above and below the critical temperature $T_{c}$ is schematically presented in the higher non-Gaussian approximation $\left(\rho^{6}\right.$ model $)$ taking into account the first confluent correction. The starting point of the problem statement in the $\mathrm{CV}$ method under study is the Hamiltonian of the 3D Ising model. After the passage to the CV set, the Jacobian of transition from the spin variables to the $\mathrm{CV}$ is calculated to ob- 


\section{V. PYLYUK}

tain a partition function similar to the Ginzburg-Landau functional. The partition function of the spin system is integrated over the layers of the $\mathrm{CV}$ phase space. The corresponding renormalization group (RG) transformation can be related to the Wilson type. Although the $\mathrm{CV}$ method as well as Wilson approach exploit the RG ideas, it is based on the use of a non-Gaussian density of measure. The main feature is the integration of shortwave spin density oscillation modes, which is generally done without using perturbation theory. The short-wave modes are characterized by the presence of the RG symmetry and are described by a non-Gaussian measure density. These modes are responsible for the formation of critical exponents and for renormalization of the coefficient of the distribution describing the long-wave modes. The calculation for long-wave modes of spin moment density oscillations is based on using the Gaussian density of measure as the basis density. The contributions to the free energy of the system from the short- and long-wave modes are calculated separately. A calculation technique for confluent corrections is elaborated in the course of determining the thermodynamic functions.

The CV method allows one to calculate the partition function of the system and to obtain not only the universal quantities (critical exponents) but also the nonuniversal characteristics. The critical region size and phase transition temperature as well as the plots of temperature dependences of the average spin moment, entropy, specific heat, and the susceptibility are obtained for different values of the interaction potential range (including the values corresponding to the nearest-neighbour interaction and the interactions between the nearest and next-nearest neighbours and between the nearest, nextnearest, and third neighbours). A nonuniversal factor determined by microscopic parameters of the system is singled out in the expressions for leading critical amplitudes and confluent correction amplitudes of the thermodynamic characteristics.

The $\rho^{6}$ model ensures a better quantitative description of the critical properties of a one-component spin system than the $\rho^{4}$ model. This follows from the results of our previous calculations (see, for example, [6]) as well as from the temperature dependences of the average spin moment $\langle\sigma\rangle$ (Fig. 9) and specific heat $C / k N$ of the 3D Ising model (Fig. 10). The calculations were made for a simple cubic lattice in zero external field with the interaction between nearest neighbours. In our calculations, we put $b=b_{\mathrm{I}}=c /(2 \sqrt{3})$. The $\rho^{6}$ model approximation includes the first confluent correction, while the approximation on the basis of the $\rho^{4}$ model takes into account the first and second confluent corrections (see $[12,41,42]$ ). The straight line 1 in Fig. 9 for the average spin moment corresponds to the $\rho^{4}$ model, line 2 to the $\rho^{6}$ model, and line 3 to the results obtained by Liu and Fisher [35] for $\tau=\left|T-T_{c}\right| / T_{c}$. The high-temperature region in Fig. 10 is presented by the curves 1,2 and 3 , while the low-temperature region by the curves $1^{\prime}, \mathscr{2}^{\prime}$ and $3^{\prime}$. The curves 1 and $t^{\prime}$ were obtained on the basis of the $\rho^{4}$ model, curves 2 and $\mathscr{2}^{\prime}$ in the $\rho^{6}$ model approximation, and curves 3 and $3^{\prime}$ correspond to the results obtained by Liu and Fisher [35]. It should be noted that the latter carried out a new numerical analysis of leading critical amplitudes of susceptibility, correlation length, specific heat, and spontaneous magnetization of 3D nearestneighbour sc, bcc, and fcc Ising models, as well as universal relations between these amplitudes. Modern estimates of the critical temperature and exponents in [35] are used in conjunction with biased inhomogeneous differential approximants to extrapolate the longest available series expansions to find the critical amplitudes. As is clearly seen from Figs. 9 and 10, the plots for the $\rho^{6}$ model agree more closely with the Liu and Fisher's results than the estimates in the $\rho^{4}$ model approximation.

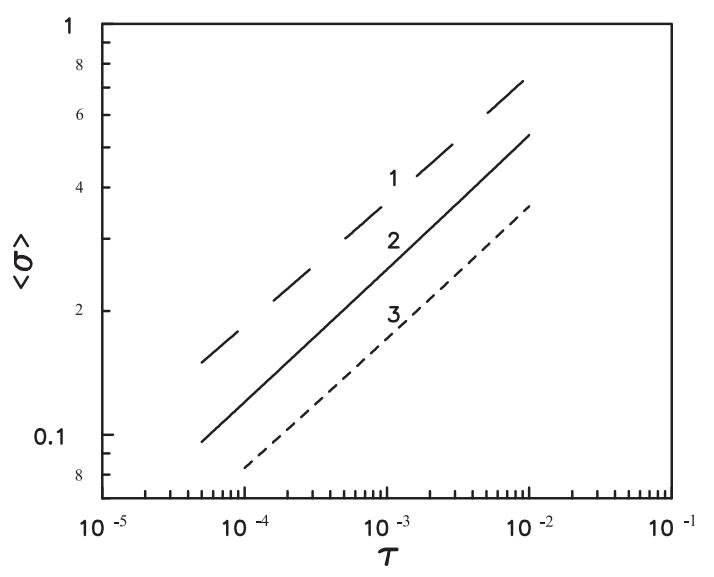

Fig. 9. Temperature dependence of the order parameter of the 3D Ising model for a simple cubic lattice. Straight line 1 corresponds to the $\rho^{4}$ model, line 2 to the $\rho^{6}$ model, and line 3 to the results obtained in [35].

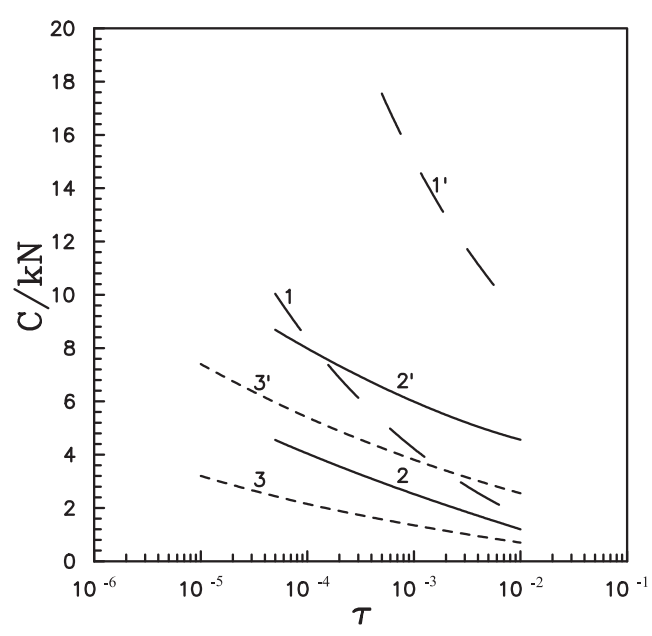

Fig. 10. Dependence of the specific heat of the system on $\tau=\left|T-T_{c}\right| / T_{c}$. Curves 1,2 and 3 correspond to $T>T_{c}$, curves $1^{\prime}, \mathscr{Q}^{\prime}$ and $\Im^{\prime}$ correspond to $T<T_{c}$. Curves 1 and $t^{\prime}$ correspond to the $\rho^{4}$ model, curves 2 and $\mathscr{2}^{\prime}$ correspond to the $\rho^{6}$ model, and curves 3 and $3^{\prime}$ correspond to the results obtained in [35]. 
The results of calculations for a $3 \mathrm{D}$ Ising system on the basis of the $\rho^{4}$ and $\rho^{6}$ models are in accord with the results obtained by other authors. For example [6], we found the critical exponents of the correlation length $\nu=0.637$, specific heat $\alpha=0.088$, the average spin moment $\beta=0.319$, the susceptibility $\gamma=1.275$, and the exponent of the first correction to scaling $\Delta_{1}=0.525$ ( $\rho^{6}$ model, $s=s^{*}$ ), as well as universal ratios of critical amplitudes of specific heat $A^{+} / A^{-}=0.435$, susceptibility $\Gamma^{+} / \Gamma^{-}=6.967$ and their combinations $\mathcal{P}=$ $\left[1-A^{+} / A^{-}\right] / \alpha=3.054, R_{c}^{+}=A^{+} \Gamma^{+} /\left[s_{0}^{3}\left(\langle\sigma\rangle^{(0)}\right)^{2}\right]=$ $0.098\left(\rho^{4}\right.$ model, $\left.s=s^{*}\right)$, where $s_{0}=\pi \sqrt{2} b / c,\langle\sigma\rangle^{(0)}$ is the critical amplitude of the average spin moment (see (23)). These estimates agree with the values $\nu=0.630$, $\alpha=0.110, \beta=0.325, \gamma=1.241, \Delta_{1}=0.498, A^{+} / A^{-}=$
$0.465, \Gamma^{+} / \Gamma^{-}=5.12, \mathcal{P}=3.90, R_{c}^{+}=0.052$ obtained by using the field-theory approach $[43-45]$ as well as with the values $\nu=0.638, \alpha=0.125, \beta=0.312, \gamma=1.250$, $\Delta_{1}=0.50, A^{+} / A^{-}=0.51, \Gamma^{+} / \Gamma^{-}=5.07, R_{c}^{+}=0.059$ calculated with the help of high-temperature expansions [46-50]. The methods existing at present make it possible to calculate universal quantities to a quite high degree of accuracy (see, for example, $[34,35,51,52]$ ). The advantage of the proposed method is the possibility of deriving analytic expressions for the phase transition temperature and the amplitudes of thermodynamic characteristics as functions of microscopic parameters of the initial system (the lattice constant and parameters of the interaction potential) that makes this method useful in describing the phase transitions in a wide class of $3 \mathrm{D}$ systems.
[1] I. R. Yukhnovskii, Dokl. Akad. Nauk SSSR 232, 312 (1977) [Sov. Phys. Dokl. 22, 18 (1977)].

[2] I. R. Yukhnovskii, Teor. Mat. Fiz. 36, 373 (1978).

[3] I. R. Yukhnovskii, Phase Transitions of the Second Order. Collective Variables Method (World Scientific, Singapore, 1987).

[4] I. R. Yukhnovs'kii, Riv. Nuovo Cimento 12, 1 (1989).

[5] Z. E. Usatenko, M. P. Kozlovskii, Phys. Rev. B 62, 9599 (2000).

[6] M. P. Kozlovskii, I. V. Pylyuk, V. V. Dukhovii, Cond. Matt. Phys. (Lviv) 11, 17 (1997).

[7] M. P. Kozlovskii, I. V. Pylyuk, V. V. Dukhovii, preprint cond-mat/9907468 (1999).

[8] M. P. Kozlovskii, Teor. Mat. Fiz. 78, 422 (1989).

[9] I. V. Pylyuk, Ukr. Fiz. Zh. (Ukr. Ed.) 41, 885 (1996).

[10] I. R. Yukhnovskii, M. P. Kozlovskii, I. V. Pylyuk, Z. Naturforsch. 46a, 1 (1991).

[11] M. P. Kozlovskii, I. V. Pylyuk, I. R. Yukhnovskii, Teor. Mat. Fiz. 87, 293 (1991).

[12] M. P. Kozlovskii, I. V. Pylyuk, I. R. Yukhnovskii, Teor. Mat. Fiz. 87, 434 (1991).

[13] M. P. Kozlovskii, I. V. Pylyuk, in: Proceedings of the All-Union Conference on Modern Problems in Statistical Physics, vol. 2, L'vov, February 3-5, 1987 [in Russian] (Naukova Dumka, Kiev, 1989), p. 50.

[14] M. P. Kozlovskii, I. V. Pylyuk, Ukr. Fiz. Zh. (Ukr. Ed.) 35, 146 (1990).

[15] V. V. Dukhovii, M. P. Kozlovskii, I. V. Pylyuk, Teor. Mat. Fiz. 107, 288 (1996).

[16] R. Guida, J. Zinn-Justin, Nucl. Phys. B 489 [FS], 626 (1997).

[17] M. M. Tsypin, Phys. Rev. Lett. 73, 2015 (1994).

[18] M. M. Tsypin, Phys. Rev. B 55, 8911 (1997).

[19] A. I. Sokolov, V. A. Ul'kov, E. V. Orlov, J. Phys. Stud. 1, 362 (1997)

[20] A. I. Sokolov, E. V. Orlov, V. A. Ul'kov, Phys. Lett. A 227, 255 (1997).

[21] M. P. Kozlovskii, I. V. Pylyuk, Z. E. Usatenko, Phys. Status Solidi B 197, 465 (1996).

[22] M. P. Kozlovskii, preprint ITP-84-35R (1984).
[23] M. P. Kozlovsky, I. V. Pylyuk, preprint ITP-85-23E (1985).

[24] S. Ma, Modern Theory of Critical Phenomena (Benjamin/Cummings, Reading (USA), 1976).

[25] I. V. Pylyuk, Teor. Mat. Fiz. 117, 442 (1998).

[26] M. P. Kozlovskii, V. V. Dukhovii, I. V. Pylyuk, Cond. Matt. Phys. (Lviv) 3, 727 (2000).

[27] M. A. Moore, D. Jasnov, M. Wortis, Phys. Rev. Lett. 22, 940 (1969).

[28] M. Ferer, M. Wortis, Phys. Rev. B 6, 3426 (1972).

[29] P. Butera, M. Comi, Phys. Rev. B 62, 14837 (2000).

[30] W. Reich, J. Phys. A 17, 3553 (1984).

[31] G. S. Pawley, R. H. Swendsen, D. J. Wallace, K. G. Wilson, Phys. Rev. B 29, 4030 (1984).

[32] A. M. Ferrenberg, D. P. Landau, Phys. Rev. B 44, 5081 (1991).

[33] D. P. Landau, A. M. Ferrenberg, J. Kor. Phys. Soc. 26, S371 (1993).

[34] D. P. Landau, Physica A 205, 41 (1994).

[35] A. J. Liu, M. E. Fisher, Physica A 156, 35 (1989).

[36] I. V. Pylyuk, Low Temp. Phys. 25, 877 (1999).

[37] I. V. Pylyuk, Low Temp. Phys. 25, 953 (1999).

[38] I. V. Pylyuk, M. P. Kozlovskii, preprint ICMP-97-06U (1997).

[39] C. Bagnuls, C. Bervillier, Phys. Rev. B 24, 1226 (1981).

[40] J. F. Nicoll, P. C. Albright, Phys. Rev. B 34, 1991 (1986).

[41] I. V. Pylyuk, M. P. Kozlovskii, Izv. Akad. Nauk SSSR, Ser. Fiz. 55, 597 (1991).

[42] M. P. Kozlovskii, I. V. Pylyuk, Phys. Status Solidi B 183, 243 (1994)

[43] J. C. Le Guillou, J. Zinn-Justin, Phys. Rev. B 21, 3976 (1980).

[44] C. Bagnuls, C. Bervillier, E. Boccara, Phys. Lett. A 103 , 411 (1984).

[45] M. Barmatz, P. C. Hohenberg, A. Kornblit, Phys. Rev. B 12, 1947 (1975).

[46] D. S. Gaunt, M. F. Sykes, J. Phys. A 6, 1517 (1973).

[47] D. M. Saul, M. Wortis, D. Jasnov, Phys. Rev. B 11, 2571 (1975).

[48] W. J. Camp, J. P. Van Dyke, Phys. Rev. B 11, 2579 
(1975).

[49] A. Aharony, P. C. Hohenberg, Phys. Rev. B 13, 3081 (1976).

[50] W. J. Camp, D. M. Saul, J. P. Van Dyke, M. Wortis,
Phys. Rev. B 14, 3990 (1976).

[51] R. Guida, J. Zinn-Justin, J. Phys. A 31, 8103 (1998).

[52] A. Pelissetto, E. Vicari, preprint cond-mat/0012164 (2000).

\title{
ТЕРМОДИНАМІЧНІ ХАРАКТЕРИСТИКИ ТРИВИМІРНИХ ІЗИНГОПОДІБНИХ СИСТЕМ ЯК ФУНКЩÏ̈ МІКРОСКОПІЧНИХ ПАРАМЕТРІВ. НАБЛИЖЕННЯ МОДЕЛІ $\rho^{6}$
}

\author{
I. В. Пилюк \\ Інститут фізики конденсованих систем Начіональной академї наук Украйни, \\ вул. Сбенціиького 1, Львіб, 79011, Украӥна
}

На основі методу колективних змінних розвинуто мікроскопічний підхід до розрахунку та дослідження термодинаміки тривимірної ізингоподібної системи вище і нижче від температури фазового переходу $T_{c}$. Математичний опис здійснено в межах вищого негаусового наближення (моделі $\rho^{6}$ ) з урахуванням поправок до скейлінгу. У виразах для основних критичних амплітуд та амплітуд поправок до скейлінгу виділено неуніверсальний множник, який залежить від мікроскопічних параметрів системи. Числові опінки розміру критичної ділянки, температури фазового переходу, а також графіки температурних залежностей ентропії та інших термодинамічних характеристик поблизу $T_{c}$ подано для різних значень радіуса ефективної дії експоненційно спадного потенціялу взаємодії. Простежено зміну вільної енергії системи в точпі фазового переходу, середнього спінового моменту та теплоємности зі зростанням відношення радіуса дії потенпіялу до сталої простої кубічної гратки. Результати розрахунків, їх зіставлення з даними інших авторів показують, що модель $\rho^{6}$, порівняно з моделлю $\rho^{4}$, забезпечує адекватніший кількісний опис критичної поведінки тривимірної ізинтоподібної системи. 